\title{
Link homology and Frobenius extensions
}

\author{
by
}

Mikhail Khovanov (New York)

\begin{abstract}
We explain how rank two Frobenius extensions of commutative rings lead to link homology theories and discuss relations between these theories, Bar-Natan theories, equivariant cohomology and the Rasmussen invariant.
\end{abstract}

Frobenius systems. Suppose $\iota: R \rightarrow A$ is an inclusion of commutative rings, and $\iota(1)=1$. The restriction functor Res $: A-\bmod \rightarrow R$-mod has left and right adjoint functors: the induction functor $\operatorname{Ind}(M)=A \otimes_{R} M$ and the coinduction functor $\operatorname{CoInd}(M)=\operatorname{Hom}_{R}(A, M)$. Following Kadison [Ka] and others, we say that $\iota$ is a Frobenius extension if the induction and coinduction functors are isomorphic. Equivalently, $\iota$ is Frobenius if the restriction functor has a biadjoint (two-sided adjoint). We note that Kadison $[\mathrm{Ka}]$ treats the more general case of not necessarily commutative $R$ and $A$. In this paper we consider only commutative rings. The following proposition is well known (or see $[\mathrm{Ka}$, Section 4]).

Proposition 1. $\iota$ is a Frobenius extension iff there exists an A-bimodule map $\Delta: A \rightarrow A \otimes_{R} A$ and an $R$-module map $\varepsilon: A \rightarrow R$ such that $\Delta$ is coassociative and cocommutative, and $(\varepsilon \otimes \mathrm{Id}) \Delta=\mathrm{Id}$.

A Frobenius extension, together with a choice of $\varepsilon$ and $\Delta$, will be denoted $\mathcal{F}=(R, A, \varepsilon, \Delta)$ and called a Frobenius system (as in $[\mathrm{Ka}]$ ).

A Frobenius system defines a 2-dimensional TQFT, a tensor functor from oriented $(1+1)$-cobordisms to $R$-modules, by assigning $R$ to the empty 1 manifold, $A$ to the circle, $A \otimes_{R} A$ to the disjoint union of two circles, etc. The structure maps $\iota, \varepsilon, \Delta, m$ (where $m$ is the multiplication in $A$ ) are assigned to basic two-dimensional cobordisms (see $[\mathrm{A}]$, [Ka], or [Kh1]).

2000 Mathematics Subject Classification: Primary 57M27.

Key words and phrases: link homology, Frobenius extensions, TQFT, equivariant cohomology. 
Each numbered formula (1), (2), etc. in this paper describes a Frobenius system. We denote the Frobenius system associated with formula $(i)$ by $\mathcal{F}_{i}=\left(R_{i}, A_{i}, \varepsilon, \Delta\right)$.

Link homology. In [Kh1] we constructed an invariant of links, based on a particular Frobenius system that we denote here by $\mathcal{F}_{1}$, with $R_{1}=\mathbb{Z}$, $A_{1}=\mathbb{Z}[X] /\left(X^{2}\right)$, the counit and comultiplication

$$
\text { (1) } \quad \varepsilon(1)=0, \quad \varepsilon(X)=1, \quad \Delta(1)=1 \otimes X+X \otimes 1, \quad \Delta(X)=X \otimes X \text {. }
$$

To a plane diagram $D$ of a link $L$ we assigned a commutative cube of $R_{1}$ modules and $R_{1}$-module homomorphisms, then passed to the total complex of the cube and took its cohomology, which ended up being independent of the choice of the diagram $D$. The construction works as well for the Frobenius system $\mathcal{F}_{2}$ with $R_{2}=\mathbb{Z}[c], A_{2}=\mathbb{Z}[X, c] /\left(X^{2}\right)$ and

$$
\begin{aligned}
\varepsilon(1) & =-c, \quad \Delta(1)=1 \otimes X+X \otimes 1+c X \otimes X, \\
\varepsilon(X) & =1, \quad \Delta(X)=X \otimes X .
\end{aligned}
$$

This system is graded, with $\operatorname{deg}(X)=2, \operatorname{deg}(c)=-2$ (we follow the grading conventions of [Kh2] rather than those of the earlier paper [Kh1]). The multiplication, comultiplication, unit and counit maps have degrees $0,2,0$ and -2 respectively. As a result, homology theories associated with this Frobenius system and with $\mathcal{F}_{1}$ are bigraded.

In a recent paper [BN] Dror Bar-Natan defined several new homology theories of links and tangles. One of his ideas, when viewed from an algebraic viewpoint, is to modify the equation $X^{2}=0$ to $X^{2}=t$, where $t$ is a formal variable (equal to one-eighth of his invariant of a closed genus 3 surface). Namely, a certain quotient of his link invariant [BN, Section 9.2] is the homology theory assigned to the Frobenius system $\mathcal{F}_{3}$ with $R_{3}=\mathbb{Z}[t]$, $A_{3}=\mathbb{Z}[X], X^{2}=t$ (i.e., $\iota: R_{3} \rightarrow A_{3}$ maps $t$ to $X^{2}$ ) and

$$
\begin{aligned}
\varepsilon(1) & =0, \quad \Delta(1)=1 \otimes X+X \otimes 1, \\
\varepsilon(X) & =1, \quad \Delta(X)=X \otimes X+t 1 \otimes 1 .
\end{aligned}
$$

This theory is graded, with $\operatorname{deg}(t)=4$. The invariant of a link is a complex of graded free $\mathbb{Z}[t]$-modules, up to chain homotopy, and its homology is a bigraded link homology theory.

Base change. Let $\mathcal{F}$ be a Frobenius system. Any homomorphism $\psi$ : $R \rightarrow R^{\prime}$ of commutative rings with $\psi(1)=1$ determines a Frobenius system $\mathcal{F}^{\prime}=\left(R^{\prime}, A^{\prime}, \varepsilon^{\prime}, \Delta^{\prime}\right)$ where $A^{\prime}=A \otimes_{R} R^{\prime}$ and the comultiplication and counit maps are induced from those for $A$ by tensoring with the identity endomorphism of $R^{\prime}$. We will say that $\mathcal{F}^{\prime}$ is obtained by base change from $\mathcal{F}$ via $\psi$. For instance, the Frobenius system $\mathcal{F}_{1}$ is obtained by base change 
(with surjective $\psi$ ) from each of $\mathcal{F}_{2}, \mathcal{F}_{3}$. Specifically, adding the relation $t=0$ to $\mathcal{F}_{3}$ recovers the $\mathcal{F}_{1}$ theory (here $\psi: \mathbb{Z}[t] \rightarrow \mathbb{Z}, \psi(t)=0$ ), while the ring homomorphism $\mathbb{Z}[t] \rightarrow \mathbb{Q}$ taking $t$ to 1 produces the Frobenius extension $\mathbb{Q} \subset \mathbb{Q}[X] /\left(X^{2}-1\right)$ leading to Lee's theory $[\mathrm{L}]$ and an application to slice genus $[\mathrm{R}]$. The specialization $t=1$ collapses the grading.

Dual system. The dual system of $\mathcal{F}$ is the Frobenius system $\mathcal{F}^{*}=$ $\left(R, A^{*}, \iota^{*}, m^{*}\right)$, where $A^{*}=\operatorname{Hom}_{R}(A, R)$ and the unit, multiplication, counit and comultiplication of $\mathcal{F}^{*}$ are obtained by dualizing the counit, comultiplication, unit and multiplication of $(R, A)$, respectively. We say that $\mathcal{F}$ is self-dual if $\mathcal{F}^{*}$ is isomorphic to $\mathcal{F}$ as an Frobenius system, i.e. there is an $R$-module isomorphism $A^{*} \cong A$ which identifies $\iota^{*}$ with $\varepsilon, m^{*}$ with $\Delta$, etc.

Proposition 2. The Frobenius systems $\left(R_{i}, A_{i}\right)$ for $i=1,2,3$ are selfdual.

This is proved by a direct computation. Note that if a system is self-dual, so are its base changes.

Twisting. Given a Frobenius system $\mathcal{F}$ and an invertible element $y \in A$, we can twist the comultiplication and counit by $y$ :

$$
\varepsilon^{\prime}(x)=\varepsilon(y x), \quad \Delta^{\prime}(x)=\Delta\left(y^{-1} x\right) .
$$

This results in a Frobenius system $\mathcal{F}^{\prime}=\left(R, A, \varepsilon^{\prime}, \Delta^{\prime}\right)$. Twisting by invertible elements of $A$ is the only way to modify the counit and comultiplication in Frobenius extensions (see [Ka, Theorem 1.6]).

To any Frobenius system $\mathcal{F}$ and a link diagram $D$ we can assign a complex of $R$-modules, denoted $\mathcal{F}(D)$, using the algorithm of [Kh1].

Proposition 3. The complexes $\mathcal{F}(D)$ and $\mathcal{F}^{\prime}(D)$ are isomorphic if $\mathcal{F}^{\prime}$ is a twisting of $\mathcal{F}$.

Proof. $\mathcal{F}(D)$ is the total complex of an $n$-cube $V_{D, \mathcal{F}}$, assigned to $D$ and $\mathcal{F}$, where $n$ is the number of crossings of $D$. At each vertex of the cube stands a tensor power of $A$, and each arrow is either a multiplication or a comultiplication map on some factors of $A^{\otimes k}$.

In fact, $V_{D, \mathcal{F}}$ and $V_{D, \mathcal{F}^{\prime}}$ are isomorphic as cubes of $R$-modules. The isomorphism is constructed by making it the identity on the source vertex of the cube and extending it arrow by arrow to the whole cube. For each vertex, the isomorphism $A^{\otimes k} \rightarrow A^{\otimes k}$ is a multiple of the identity map, with the coefficient being a product of powers of $y_{i}^{-1}, 1 \leq i \leq k$, where

$$
y_{i}=1 \otimes \cdots \otimes 1 \otimes y \otimes 1 \otimes \cdots \otimes 1 \in A^{\otimes k} .
$$

This isomorphism of cubes induces an isomorphism of the complexes $\mathcal{F}(D)$ and $\mathcal{F}^{\prime}(D)$. 
Corollary 1. If $\mathcal{F}^{\prime}$ is a twisting of $\mathcal{F}$ then $\mathrm{H}(D, \mathcal{F}) \cong \mathrm{H}\left(D, \mathcal{F}^{\prime}\right)$.

Let us look at the $\mathcal{F}_{2}$ theory from this viewpoint. The element $1+c X$ in $A_{2}$ is invertible, $(1+c X)(1-c X)=0$. The twisting $\mathcal{F}_{2}^{\prime}$ of $\mathcal{F}_{2}$ by this element is a Frobenius system with the counit and comultiplication given by formula (1). Therefore, $\mathcal{F}_{2}^{\prime}$ is just the Frobenius system $\mathcal{F}_{1}$ with the ground ring extended by adding a free variable $c$.

Corollary 2. For any link $L$ and its diagram $D$ the complexes $\mathcal{F}_{1}(D)$ $\otimes_{\mathbb{Z}} \mathbb{Z}[c]$ and $\mathcal{F}_{2}(D)$ are isomorphic. Consequently, there is an isomorphism of cohomology groups

$$
\mathrm{H}\left(L, \mathcal{F}_{2}\right) \cong \mathrm{H}\left(L, \mathcal{F}_{1}\right) \otimes_{\mathbb{Z}} \mathbb{Z}[c] .
$$

Therefore, adding $c$ does not provide new information. Moreover, Jacobsson $[\mathrm{J}]$ showed that the $\mathcal{F}_{2}$ theory is not invariant under cobordisms. Multiplication by $1+c X$ appears in his work as well.

Thus, twisting preserves link homology groups but not, in general, link homology functors.

Invariance under the first Reidemeister move. We would like to know for which Frobenius systems $\mathcal{F}$ the complexes $\mathcal{F}\left(D_{1}\right)$ and $\mathcal{F}\left(D_{2}\right)$ are chain homotopy equivalent whenever $D_{1}, D_{2}$ are related by a Reidemeister move. Any such system produces a homology theory of links (where to a link $L$ we assign the homology $\mathrm{H}(D, \mathcal{F})$ of the complex $\mathcal{F}(D)$ for a diagram $D$ of $L)$.

The complex assigned to a one-crossing knot diagram is either

$$
0 \rightarrow A \otimes_{R} A \stackrel{m}{\rightarrow} A \rightarrow 0
$$

or

$$
0 \rightarrow A \stackrel{\Delta}{\rightarrow} A \otimes_{R} A \rightarrow 0
$$

depending on the crossing's sign. Each of these complexes must be chain homotopy equivalent to $0 \rightarrow A \rightarrow 0$, via an $R$-linear, and, preferably, $A$ linear homotopy. The unit map $\iota \otimes$ Id is a section of $m$, and the first complex decomposes as a sum of a contractible complex and $0 \rightarrow \operatorname{ker}(m) \rightarrow 0$. Hence, we need an $A$-module isomorphism $\operatorname{ker}(m) \cong A$. Since

$$
\operatorname{ker}(m) \cong \operatorname{coker}(\iota) \otimes_{R} A \cong(A / R) \otimes_{R} A
$$

(where $A / R$ is the $R$-module quotient), the invariance follows if $A / R$ is a free $R$-module of rank 1 . Assuming this and pulling back $1 \in R \cong A / R$ to $A$ we get $X \in A$ such that $A \cong R 1 \oplus R X$ as $R$-modules.

We say that $\mathcal{F}$ has rank two if there exists $X \in A$ such that $A \cong R 1 \oplus R X$. 
A universal rank two Frobenius system. Let $\mathcal{F}_{4}$ be the Frobenius system with

$$
R_{4}=\mathbb{Z}[a, c, e, f, h, t] /(a e-c f, a f+c h f-c e t-1)
$$

and $A_{4}=R_{4}[X] /\left(X^{2}-h X-t\right)$. The comultiplication and counit are

$$
\begin{aligned}
\Delta(1) & =(e t-h f) 1 \otimes 1+e X \otimes X+f(1 \otimes X+X \otimes 1), \\
\Delta(X) & =f t 1 \otimes 1+e t(1 \otimes X+X \otimes 1)+(f+e h) X \otimes X, \\
\varepsilon(1) & =-c, \quad \varepsilon(X)=a .
\end{aligned}
$$

$R_{4}$ and $A_{4}$ are graded, with $a, c, e, f, h, t, X$ in degrees $0,-2,-2,0,2,4,2$ respectively.

Proposition 4. $\mathcal{F}_{4}$ is a rank two graded Frobenius system, universal in the following sense. Suppose $\mathcal{F}^{\prime}$ is a rank two Frobenius system, and $X^{\prime} \in A^{\prime}$ a splitting element, $A^{\prime} \cong R^{\prime} 1 \oplus R^{\prime} X^{\prime}$. There exists a unique homomorphism $\psi: A_{4} \rightarrow A^{\prime}$ with $\psi(X)=X^{\prime}$ that realizes $\mathcal{F}^{\prime}$ as a base change of $\mathcal{F}_{4}$.

Proof. That $\mathcal{F}_{4}$ is a graded rank two Frobenius system can be verified by a direct computation. Furthermore, for $X^{\prime}$ as above, we have $X^{\prime 2}=h^{\prime} X^{\prime}+t^{\prime}$, $\varepsilon^{\prime}(1)=-c^{\prime}$ and $\varepsilon^{\prime}\left(X^{\prime}\right)=a^{\prime}$ for unique $h^{\prime}, t^{\prime}, a^{\prime}, c^{\prime} \in R^{\prime}$. Write

$$
\Delta^{\prime}(1)=d^{\prime} 1 \otimes 1+e^{\prime} X^{\prime} \otimes X^{\prime}+f^{\prime}\left(1 \otimes X^{\prime}+X^{\prime} \otimes 1\right) .
$$

Since $\Delta^{\prime}$ is a map of $A^{\prime}$-bimodules,

$$
\left(X^{\prime} \otimes 1\right) \Delta^{\prime}(1)=\left(1 \otimes X^{\prime}\right) \Delta^{\prime}(1),
$$

implying $d^{\prime}=e^{\prime} t^{\prime}-h^{\prime} f^{\prime}$. Define $\psi$ by taking generators $a, c, e, f, h, t, X$ of $A_{4}$ to the corresponding elements of $A^{\prime}$.

REMARK. This system has a geometric description in terms of dotted surfaces (where a dot stands for multiplication by $X$ ). For instance, the invariant of a sphere decorated by a dot is $\varepsilon(X)=a$. The relation $X^{2}=$ $h X+t$ translates into the following skein relation: a disk decorated by two dots equals a disk decorated by a single dot times $h$ plus a disk times $t$. The formula for $\Delta(1)$ translates into a surgery skein relation for a tube (compare with [Kh3] and [BN, Section 11.2]). Adding a dot to a surface corresponds to taking the connected sum of the surface with the torus and dividing by 2 , in the language of Bar-Natan [BN].

Since $f+e X$ is invertible, with inverse $a+c h-c X$, and has degree 0 , we can twist by it. The comultiplication and counit become

$$
\begin{aligned}
\varepsilon(1) & =0, \quad \Delta(1)=1 \otimes X+X \otimes 1-h 1 \otimes 1, \\
\varepsilon(X) & =1, \quad \Delta(X)=X \otimes X+t 1 \otimes 1 .
\end{aligned}
$$

After the twist, all the structure maps depend on $h$ and $t$ only. Let $\mathcal{F}_{5}$ be 
the Frobenius system with

$$
R_{5}=\mathbb{Z}[h, t], \quad A_{5}=R_{5}[X] /\left(X^{2}-h X-t\right), \quad \operatorname{deg}(h)=2, \quad \operatorname{deg}(t)=4,
$$

and $\varepsilon, \Delta$ given by (5). We have

Proposition 5. Any rank two Frobenius system is obtained from $\mathcal{F}_{5}$ by a composition of a base change and a twist.

There is a map from Bar-Natan's theory $[\mathrm{BN}]$ to $\mathcal{F}_{5}$ given by introducing dotted surfaces and skein relations between them. The coefficients of skein relations are the structure constants of $\mathcal{F}_{5}$. The decomposition of $\Delta(1)$ in formula (5) becomes a skein relation for dotted surfaces that implies the $4 T u$ relation of Bar-Natan. Although this map from Bar-Natan's theory to $\mathcal{F}_{5}$ is neither surjective nor injective already for the empty link, his arguments are universal, being instantly adoptable to $\mathcal{F}_{5}$ and any base change of the latter. The next result follows from $[\mathrm{BN}]$ at once.

Proposition 6. The complexes $\mathcal{F}_{5}\left(D_{1}\right)$ and $\mathcal{F}_{5}\left(D_{2}\right)$ are isomorphic if $D_{1}, D_{2}$ are two diagrams of the same oriented link. The Frobenius system $\mathcal{F}_{5}$ determines a bigraded link homology theory which is functorial for link cobordisms up to sign indeterminacy. Any (graded) Frobenius system obtained by a (graded) base change from $\mathcal{F}_{5}$ determines a (bi) graded homology theory of links, functorial for link cobordisms up to sign indeterminacy.

In view of Proposition 5, any rank two Frobenius system (after twisting, if necessary) produces a cohomology theory of links. On the chain level, all the information is already captured by $\mathcal{F}_{5}$, since $\mathcal{F}^{\prime}(D) \cong \mathcal{F}_{5}(D) \otimes_{R} R^{\prime}$ for any $\mathcal{F}^{\prime}$ given by a base change from $\mathcal{F}_{5}$.

Bar-Natan's theory [BN] cannot be immediately expressed in the language of Frobenius systems. Indeed, for Frobenius systems the homology of 2-component unlink is $A \otimes_{R} A$, the second tensor power of the unknot's homology $A$ over the homology $R$ of the empty knot. In Bar-Natan's framework there is no such isomorphism. In particular, a tube (which should represent $\Delta(1))$ cannot be decomposed into a union of surfaces separating the two boundaries of the tube.

ExAmples. 1 . The quotient of $\mathcal{F}_{5}$ by the ideal $(h)$ produces the Frobenius system $\mathcal{F}_{3}$ described earlier. The variable $h$ can also be removed by $X \rightarrow X-h / 2$ if 2 is made invertible in the ground ring (at the cost of modifying $t$ ).

2. The Frobenius system $\mathcal{F}_{5}$ is almost self-dual. Namely, the dual of $\mathcal{F}_{5}$ is the system where $h$ is changed to $-h$ in the structure maps. In particular, any base change $\psi$ of $\mathcal{F}_{5}$ with $\psi(2 h)=0$ is self-dual. Dual Frobenius systems make an appearance in knot homology, for there is an isomorphism of chain complexes 


$$
C\left(D^{!}, \mathcal{F}\right) \cong C\left(D, \mathcal{F}^{*}\right)^{*}
$$

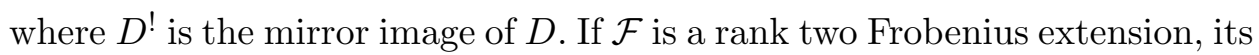
dual also has rank two, and the above equation descends to an isomorphism of link invariants.

3. Link homology theory discovered by Bar-Natan in [BN, Section 9.3] and investigated by Turner $[\mathrm{T}]$ is given by a Frobenius system $\mathcal{F}_{6}$ with

$$
R_{6}=\mathbb{F}_{2}[H], \quad A_{6}=R_{6}[X] /\left(X^{2}-H X\right), \quad \operatorname{deg}(H)=2,
$$

where $\mathbb{F}_{2}$ is the 2-element field, and

$$
\begin{aligned}
\varepsilon(1) & =0, \quad \Delta(1)=1 \otimes X+X \otimes 1+H 1 \otimes 1, \\
\varepsilon(X) & =1, \quad \Delta(X)=X \otimes X .
\end{aligned}
$$

This system is self-dual. The base change $\psi: R_{5} \rightarrow R_{6}$ to this system is given by $\psi(h)=H, \psi(t)=0$.

4. Field extensions. Any field extension $R \subset A$ of finite degree is Frobenius, and any nonzero $R$-linear map $A \rightarrow R$ can serve as a counit $\varepsilon$. In particular, any degree two field extension $R \subset A$ gives rise to a link homology theory. We do the base change $R \rightarrow \bar{R}$, where $\bar{R}$ is the algebraic closure of $R$. This base change preserves the dimension of homology groups (as $R$-, respectively, $\bar{R}$-vector spaces):

$$
\operatorname{dim}_{R} \mathrm{H}(L, \mathcal{F})=\operatorname{dim}_{\bar{R}} \mathrm{H}(L, \overline{\mathcal{F}}),
$$

where $\overline{\mathcal{F}}$ is the above base change of $\mathcal{F}=(R, A, \varepsilon, \Delta)$. Let $\bar{A}=A \otimes_{R} \bar{R}$. There are two cases to consider.

(a) The extension $R \subset A$ is separable. Then $\bar{A} \cong \bar{R} \times \bar{R}$, as an $\bar{R}$-algebra, and the resulting theory is the one studied by Lee $[\mathrm{L}]$ in characteristic 0 , and by Shumakovitch [Sh] in finite characteristic, with

$$
\operatorname{dim}_{\bar{R}} \mathrm{H}(L, \overline{\mathcal{F}})=2^{m},
$$

where $m$ is the number of components of $L$. In particular, the total rank of homology groups $\mathrm{H}(L, \mathcal{F})$ depends only on $m$.

(b) The extension $R \subset A$ is inseparable. Then $\operatorname{char} R=2$ and $A \cong$ $R[y] /\left(y^{2}+t\right)$ where $t \in R, \sqrt{t} \notin R$. We have $\bar{A} \cong \bar{R}[X] /\left(X^{2}\right)$ where $X=$ $y+\sqrt{t}$, and $\sqrt{t} \in \bar{R}$. Twisting $\bar{\varepsilon}$, we can assume $\bar{\varepsilon}(1)=0, \bar{\varepsilon}(X)=1$. The resulting theory $\overline{\mathcal{F}}$ is obtained from $\mathcal{F}_{1}$ by the base change

$$
\mathbb{Z} \rightarrow \mathbb{F}_{2} \rightarrow \bar{R}
$$

The intermediate theory (with $R=\mathbb{F}_{2}$ ) is simply the original theory of [Kh1, Section 7] with coefficients in the 2-element field. Denote it by $\mathrm{H}\left(L, \mathbb{F}_{2}\right)$. We have

$$
\operatorname{dim}_{R} \mathrm{H}(L, \mathcal{F})=\operatorname{dim}_{\mathbb{F}_{2}} \mathrm{H}\left(L, \mathbb{F}_{2}\right)
$$

for any $\mathcal{F}$ given by an inseparable degree two field extension $R \subset A$. 
Equivariant cohomology. The Frobenius extension $\mathcal{F}_{1}$ has a cohomological interpretation: $R_{1} \cong \mathbb{Z}$ is isomorphic to the cohomology ring of a point, and $A_{1} \cong \mathbb{Z}[X] /\left(X^{2}\right)$ to the cohomology ring of a 2 -sphere. The trace map $\varepsilon$ is the integration along the fundamental cycle on $\mathbb{S}^{2}$.

Other extensions that we considered have similar interpretations via equivariant cohomology. Suppose that a topological group $G$ acts continuously on $\mathbb{S}^{2}$. Define

$$
R_{G} \cong \mathrm{H}_{G}^{*}(p, \mathbb{Z})=\mathrm{H}^{*}(B G, \mathbb{Z})
$$

to be the $G$-equivariant cohomology ring of a point $p$ (where $B G$ is the classifying space of $G$ ), and

$$
A_{G} \cong \mathrm{H}_{G}^{*}\left(\mathbb{S}^{2}, \mathbb{Z}\right)=\mathrm{H}^{*}\left(\mathbb{S}^{2} \times_{G} E G, \mathbb{Z}\right)
$$

the equivariant cohomology ring of the 2-sphere. Then, in several cases, $\left(R_{G}, A_{G}\right)$ is a rank 2 Frobenius extension, with $\varepsilon$ induced by integration along the fibers of the $\mathbb{S}^{2}$-fibration

$$
\mathbb{S}^{2} \times_{G} E G \rightarrow B G .
$$

EXAmples. 1. The standard action of $G=S U(2)$ on $\mathbb{C}^{2}$ induces an action on $\mathbb{S}^{2}$ (with $-I$ acting trivially) and leads to the Frobenius system $\mathcal{F}_{3}$. Indeed,

$$
\begin{aligned}
& R_{3}=\mathbb{Z}[t] \cong \mathrm{H}^{*}(B S U(2), \mathbb{Z})=\mathrm{H}^{*}\left(\mathbb{H} \mathbb{P}^{\infty}, \mathbb{Z}\right), \\
& A_{3}=\mathbb{Z}[X] \cong \mathrm{H}^{*}\left(\mathbb{S}^{2} \times_{S U(2)} E S U(2), \mathbb{Z}\right) \cong \mathrm{H}^{*}\left(\mathbb{C} \mathbb{P}^{\infty}, \mathbb{Z}\right), \quad X^{2}=t .
\end{aligned}
$$

$X$ here is the two-dimensional cohomology class of $\mathbb{C P}^{\infty}$ which evaluates to 1 on $\mathbb{C P}^{1} \subset \mathbb{C P}^{\infty}$. We choose $t \in \mathrm{H}^{4}\left(\mathbb{H} \mathbb{P}^{\infty}, \mathbb{Z}\right)$ so that its pullback to $\mathbb{C P}^{\infty}$ equals $X^{2}$. Also, $\varepsilon(X)=1$. The geometric counterpart of the $S U(2)$ equivariant theory was considered by Seidel and Smith [S].

2. Taking $G$ to be the group $U(2)$ with the usual action on $\mathbb{S}^{2}$ (so that the center $U(1)$ acts trivially), we get the Frobenius system $\mathcal{F}_{5}$ :

$$
\begin{aligned}
& R_{5}=\mathbb{Z}[h, t] \cong \mathrm{H}^{*}(B U(2), \mathbb{Z}) \cong \mathrm{H}(\mathrm{Gr}(2, \infty), \mathbb{Z}), \\
& A_{5}=\mathbb{Z}[h, X] \cong \mathrm{H}^{*}\left(\mathbb{S}^{2} \times_{U(2)} E U(2), \mathbb{Z}\right) \cong \mathrm{H}^{*}(B U(1) \times B U(1), \mathbb{Z}) .
\end{aligned}
$$

$\operatorname{Gr}(2, \infty)$ is the Grassmannian of complex planes $\mathbb{C}^{2}$ in $\mathbb{C}^{\infty}$, its cohomology ring is freely generated by $h$ and $t$ in degrees 2 and 4 , while $B U(1) \cong \mathbb{C P}^{\infty}$. Notice that $A_{5}$ is the polynomial ring with generators $X, Y=h-X$, and $R_{5}$ is the ring of symmetric functions in $X$ and $Y$, with $h$ and $-t$ the elementary symmetric functions.

3. $G=U(1)$, the group of rotations of $\mathbb{S}^{2}$ about a fixed axis. In this case we get a Frobenius system $\mathcal{F}_{7}$ which is the quotient of $\mathcal{F}_{5}$ by the ideal $(t)$ : 


$$
\begin{aligned}
R_{7} & =\mathbb{Z}[h], & A_{7} & =\mathbb{Z}[X, h] /\left(X^{2}-h X\right), \\
\varepsilon(1) & =0, & \Delta(1) & =1 \otimes X+X \otimes 1-h 1 \otimes 1, \\
\varepsilon(X) & =1, & \Delta(X) & =X \otimes X,
\end{aligned}
$$

The system $\mathcal{F}_{6}$ is the modulo 2 specialization of $\mathcal{F}_{7}$ and can be described via equivariant $U(1)$-cohomology with coefficients in $\mathbb{F}_{2}$.

If we change the coefficient ring of equivariant cohomology from $\mathbb{Z}$ to any field $k$ of characteristic other than 2, then the substitution $X \rightarrow X-h / 2$ takes us to the theory which is a base change of $\mathcal{F}_{3}$ (the latter also considered over $k$ ) with $t=-h^{2} / 4$. The homology of the resulting theory is given by suitably doubling (with a shift) the $\mathcal{F}_{3}$ homology (over $k$ ).

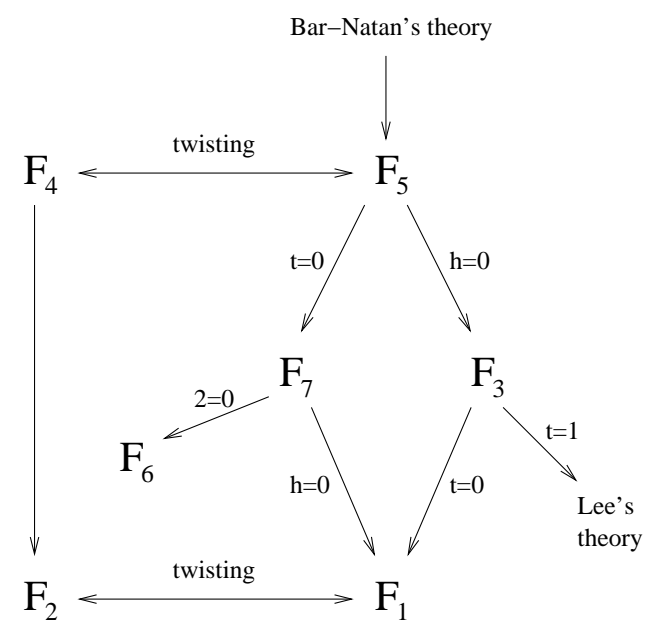

Fig. 1. A diagram of link homology theories

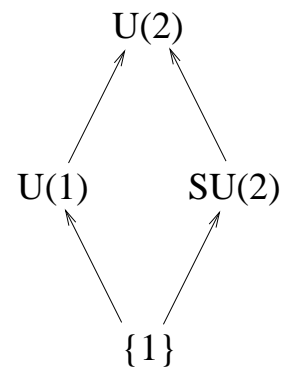

Fig. 2. Some subgroups of $U(2)$

Each of the four theories $\mathcal{F}_{1}, \mathcal{F}_{3}, \mathcal{F}_{5}, \mathcal{F}_{7}$ at the vertices of the central rombus in Figure 1 is the $G$-equivariant theory for some connected closed subgroup of $U(2)$ (see Figure 2). Group inclusion arrows in Figure 2 are reversals of base change arrows in Figure 1. 
Bar-Natan's theory and the Rasmussen invariant. To a diagram $D$ of an oriented link $L$ we can associate the complex $\mathcal{F}_{3}(D)$ of graded free $\mathbb{Z}[t]$-modules (recall that $t$ is one-eighth of the closed genus three surface in $[\mathrm{BN}]$, as well as a generator of $\left.\mathrm{H}^{4}\left(\mathbb{H}^{\infty}, \mathbb{Z}\right)\right)$. The chain homotopy equivalence class of this complex is an invariant of $L$. Denote the complex $\mathcal{F}_{3}(D) \otimes_{\mathbb{Z}} \mathbb{Q}$ of graded free $\mathbb{Q}[t]$-modules by $C_{t}(D)$ and its cohomology by $\mathrm{H}_{t}(D)$ (this is exactly Bar-Natan's universal theory over $\mathbb{Q}$ ). The cohomology $\mathrm{H}_{t}(D)$ is a finitely generated graded $\mathbb{Q}[t]$-module and thus a direct sum of torsion modules $\mathbb{Q}[t] /\left(t^{m}\right)$, for various $m$, and free modules $\mathbb{Q}[t]$. Denote by $\operatorname{Tor}(D)$ the torsion submodule of $\mathrm{H}_{t}(D)$ and by $\mathrm{H}^{\prime}(D)$ the quotient $\mathrm{H}_{t}(D) / \operatorname{Tor}(D)$. We denote the corresponding link homologies by $\operatorname{Tor}(L)$ and $\mathrm{H}^{\prime}(L)$, respectively. Both of these theories are cobordism-friendly: given a link cobordism $S$ from $L_{1}$ to $L_{2}$ there are well defined (up to overall sign) homomorphisms

$$
\operatorname{Tor}(S): \operatorname{Tor}\left(L_{1}\right) \rightarrow \operatorname{Tor}\left(L_{2}\right), \quad \mathrm{H}^{\prime}(S): \mathrm{H}^{\prime}\left(L_{1}\right) \rightarrow \mathrm{H}^{\prime}\left(L_{2}\right) .
$$

To define $\mathrm{H}^{\prime}(S)$ on an element $\alpha \in \mathrm{H}^{\prime}\left(L_{1}\right)$, pull $\alpha$ back to $\mathrm{H}\left(L_{1}\right)$ and apply the homomorphism $\mathrm{H}_{t}(S)$ composed with the quotient map $\mathrm{H}_{t}\left(L_{2}\right) \rightarrow$ $\mathrm{H}^{\prime}\left(L_{2}\right)$. The map $\operatorname{Tor}(S)$ is simply the restriction of $\mathrm{H}_{t}(S)$ to the torsion submodule of $\mathrm{H}\left(L_{1}\right)$.

Proposition 7. $\mathrm{H}^{\prime}(L)$ is a free $\mathbb{Q}[t]$-module of rank $2^{m}$, where $m$ is the number of components of $L$.

Proof. This follows from Lee [L]. The quotient of the complex $C_{t}(D)$ by its subcomplex $(t-1) C_{t}(D)$ has cohomology of dimension equal to the rank of $\mathrm{H}^{\prime}(L)$.

Assume now $L$ is a knot. Then $C_{t}(D)$ is naturally a complex of free $\mathbb{Q}[X]$-modules, where $X^{2}=t$.

Proposition $8 \cdot \mathrm{H}^{\prime}(L)$ is a free $\mathbb{Q}[X]$-module of rank one concentrated in cohomological degree 0.

Therefore, as a graded $\mathbb{Q}[X]$-module, $\mathrm{H}^{\prime}(L) \cong \mathbb{Q}[X]\left\{-s^{\prime}(L)-1\right\}$ for some even integer $s^{\prime}(L)$. Notice that $\mathrm{H}_{t}(L)$ is nontrivial only in odd $q$-degrees, since $L$ is a knot, hence the shift is by an odd degree. It is clear from the definition that $s^{\prime}(L)$ is the Rasmussen invariant $s(L)$, assuming that we normalize as in $[\mathrm{Kh} 2,4]$.

The quotient of $\mathrm{H}^{\prime}(L)$ by the ideal $(t-1)$ gives Lee's theory. In the latter any connected knot cobordism induces a nontrivial homomorphism (see $[\mathrm{R}]$ ). Lifting to $\mathrm{H}^{\prime}(L)$, we have

Proposition 9. Any connected genus $g$ cobordism $S$ between knots $L_{1}$ and $L_{2}$ induces a nonzero grading-preserving map

$$
\mathrm{H}^{\prime}(S): \mathrm{H}^{\prime}\left(L_{1}\right) \rightarrow \mathrm{H}^{\prime}\left(L_{2}\right)\{-2 g\} .
$$


All other results of Rasmussen [R] admit a natural interpretation via $H^{\prime}$ as well. The Frobenius system $\mathcal{F}_{3}$ allows working with graded rather than filtered complexes, making Rasmussen structures slightly more explicit and bundling up a number of invariance results from several papers into that for $\mathcal{F}_{3}$ implied by Bar-Natan $[\mathrm{BN}]$.

The complex $C_{t}(D) / t C_{t}(D)$ is the original complex of [Kh1, Section 7] with coefficients in $\mathbb{Q}$, which we denote $C(D)$. Since $\mathbb{Q}[X]$ has homological dimension 1, the complex $C_{t}(D)$ is isomorphic to the direct sum of complexes

$$
0 \rightarrow \mathbb{Q}[X]\{2 m+i\} \stackrel{X^{m}}{\rightarrow} \mathbb{Q}[X]\{i\} \rightarrow 0
$$

for various $m>0, i \in \mathbb{Z}$, a contractible complex, and the complex

$$
0 \rightarrow \mathbb{Q}[X]\{-s(L)-1\} \rightarrow 0 .
$$

Reducing modulo $t$, we see that $C(D)$ is isomorphic, modulo contractible complexes, to the direct sum of

$$
0 \rightarrow A\{2 m+i\} \stackrel{0}{\rightarrow} A\{i\} \rightarrow 0,
$$

for $m>1$,

$$
0 \rightarrow A\{2+i\} \stackrel{X}{\rightarrow} A\{i\} \rightarrow 0
$$

( $m=1$ case), and the complex $0 \rightarrow A\{-s(L)-1\} \rightarrow 0$. Here $A=$ $\mathbb{Q}[X] /\left(X^{2}\right)$.

In particular, complexes

$$
0 \rightarrow A \stackrel{X}{\rightarrow} A \stackrel{X}{\rightarrow} \cdots \stackrel{X}{\rightarrow} A \rightarrow 0
$$

of length greater than 1 (i.e. with more than two $A$ 's) cannot appear as direct summands of $C(D)$, which settles one of the problems implicitly raised in [Kh4, Section 3]. Thus, the rank of the reduced homology $\widetilde{\mathrm{H}}(L)$ is always less than the rank of $\mathrm{H}(L)$, with the difference $\operatorname{dim} \mathrm{H}(L)-\operatorname{dim} \widetilde{\mathrm{H}}(L)-1$ being twice the number of terms in $C_{t}(D)$ of the form $(*)$ for $m>1$.

Acknowledgments. I am grateful to Dror Bar-Natan and Dmitry Fuchs for enlightening discussions. Many useful suggestions from the referee were incorporated into the final version of the paper. While writing this paper, I was partially supported by the NSF grant DMS-0407784.

\section{References}

[A] L. Abrams, Two dimensional topological quantum field theories and Frobenius algebras, J. Knot Theory Ramif. 5 (1996), 569-587.

[BN] D. Bar-Natan, Khovanov's homology for tangles and cobordisms, math.GT/0410495.

[J] M. Jacobsson, Khovanov's conjecture over $\mathbb{Z}[c]$, math.GT/0308151. 
[Ka] L. Kadison, New examples of Frobenius extensions, University Lecture Series 14, Amer. Math. Soc., 1999.

[Kh1] M. Khovanov, A categorification of the Jones polynomial, Duke Math. J. 101 (2000), 359-426.

[Kh2] -, A functor-valued invariant of tangles, Algebr. Geom. Topol. 2 (2002), 665-741.

[Kh3] -, sl(3) link homology I, ibid. 4 (2004) 1045-1081.

[Kh4] -, Patterns in knot cohomology I, Experiment. Math. 12 (2003), 365-374.

[L] E. S. Lee, An endomorphism of the Khovanov invariant, math.GT/0210213, Adv. Math., to appear.

[R] J. Rasmussen, Khovanov homology and the slice genus, math.GT/0402131.

[Sh] A. Shumakovitch, Torsion of the Khovanov homology, math.GT/0405474.

[S] I. Smith, Talk at MSRI, March 2004.

[T] P. Turner, Calculating Bar-Natan's characteristic two Khovanov homology, math.GT/0411225.

Department of Mathematics

Columbia University

New York, NY 10027, U.S.A.

E-mail: khovanov@math.columbia.edu

Received 24 December 2004;

in revised form 22 October 2005 